\title{
Ocular onchocerciasis: current management and future prospects
}

This article was published in the following Dove Press journal:

Clinical Ophthalmology

I4 October 201 I

Number of times this article has been viewed

\section{Olufemi Emmanuel Babalola \\ Department of Ophthalmological Surgery, Bingham University Teaching Hospital, New Karu, Nassarawa State, Nigeria}

\begin{abstract}
This paper reviews the current management of onchocerciasis and its future prospects. Onchocerciasis is a disease affecting millions of people in Africa, South and Central America, and Yemen. It is spread by the blackfly as a vector and caused by the filarial nematode, Onchocerca volvulus. A serious attempt was made by the Onchocerciasis Control Program between 1975 and 2002 to eliminate the vector in eleven of the endemic countries in West Africa, and with remarkable success. Formerly, the treatment was with diethyl carbamazine for the microfilaria and suramin for the adult worm. These drugs are now known to be toxic and unsuitable for mass distribution. In particular, they precipitate optic nerve disease. With the discovery of ivermectin, a much safer microfilaricide, and the decision of Merck to distribute the drug free of charge for as long as needed, the strategy of control switched to mass drug administration through community-directed treatment with ivermectin. So far, millions have received this annual or biannual treatment through the African Program for Onchocerciasis Control and the Onchocerciasis Elimination Program for the Americas. However, the problem with ivermectin is that it is a monotherapy microfilaricide which has limited effect on the adult worm, and thus will need to be continued for the life span of the adult worm, which may last up to 15 years. There are also early reports of resistance. Serious encephalopathy and death may occur when ivermectin is used in subjects heavily infested with loiasis. It seems unlikely that a break in transmission will occur with community-directed treatment with ivermectin in Africa because of population migrations and the highly efficient vector, but in the Americas some countries such as Columbia and the Oaxaca focus in Mexico have reported eradication. Vector control is only now applicable in selected situations, and particularly to control the nuisance value of the blackfly. Trials are ongoing for alternatives to ivermectin. Candidate drugs include moxidectin, a macrofilaricide, doxycycline which targets the Wolbachia endosymbiont, and flubendazole, which shows promise with the newer oral cyclodextrin formulation.
\end{abstract}

Keywords: onchocerciasis, river blindness, ocular, management

\section{Introduction}

The management of onchocerciasis is challenging. Firstly, it has to be carried on at both individual and community levels. Secondly, considerations have to be given to measures against the vector, ie, the Simulidae species. Thirdly, the ideal treatment of the infected individual should be directed at both the adult worm and the microfilaria. Finally, treatment has to be directed at skin and ocular disease. Invariably the patient with skin disease has eye disease and vice versa, and has to be treated holistically.

Onchocerciasis, also known as river blindness, is an infection caused by the filarial nematode, Onchocerca volvulus. ${ }^{1,2}$ It is transmitted by various species of Simulidae,
Babalola

Department of Surgery, Bingham

University, New Karu, Nassarawa State,

Nigeria

Tel +2348053693682

Fax +23493148307

Email bablo57@yahoo.com 
known as the blackfly. ${ }^{3}$ It is a problem of major social and health significance in various countries of sub-Saharan Africa, South and central America, and in some countries in the Arabian Peninsula, notably Yemen. ${ }^{1}$

In 1995, the World Health Organization (WHO) estimated that 122.9 million people worldwide were at risk of infection, of whom approximately 17.7 million were infected and about 300,000 were blind. ${ }^{4}$ More recent estimates have been refined by widespread use of rapid epidemiological mapping of onchocerciasis methods. In a recent estimate published by Amazigo et al, it was suggested that within the African Program for Onchocerciasis Control (APOC) countries, there are 87 million people at risk, of whom 37 million are infected, 300,000 are blind, and a further 500,000 are visually impaired. ${ }^{5}$ The Onchocerciasis Control Project (OCP) was carried out in Benin, Burkina Faso, Côte d'Ivoire, Ghana, Mali, Niger, Sierra Leone, Guinea Republic, Guinea Bissau, Senegal, and Togo. The non-OCP countries now within the APOC project are African countries which also have endemic onchocerciasis but were not included in the original OCP area. These include Liberia (which had been left out largely due to internal conflict), Cameroon, Chad, the Democratic Republic of Congo, Ethiopia, Malawi, Nigeria, the Republic of Congo, Sudan, Tanzania, Uganda, Rwanda, Burundi, the Central African Republic, Gabon, and Equatorial Guinea (see Figures 1 and 2 for a map of the OCP and APOC areas). Figure 3 shows the countries covered by the Onchocerciasis Elimination Program for the Americas.

Compared with the same statistics put out by WHO in 1987, the present figures appear to suggest that measures taken to control the transmission of onchocerciasis in recent times, including the OCP and the ivermectin distribution program, have had little impact on the overall number of the blind and visually impaired victims of onchocerciasis, ${ }^{6}$ and it is estimated that worldwide, 78 million are at risk, 17 million are infected, and over 300,000 are blind. The latest figures are probably more precise because rapid epidemiological mapping of onchocerciasis has been carried out in several countries.

\section{Dermatologic and ocular manifestations}

Human involvement includes both dermatologic and ocular disease. Skin manifestations have been well described by Murdoch et al. ${ }^{7}$ These include acute and chronic papular onchodermatitis, scratch marks, lichenification, and typical pigmentary skin changes known as leopard skin. Major ocular

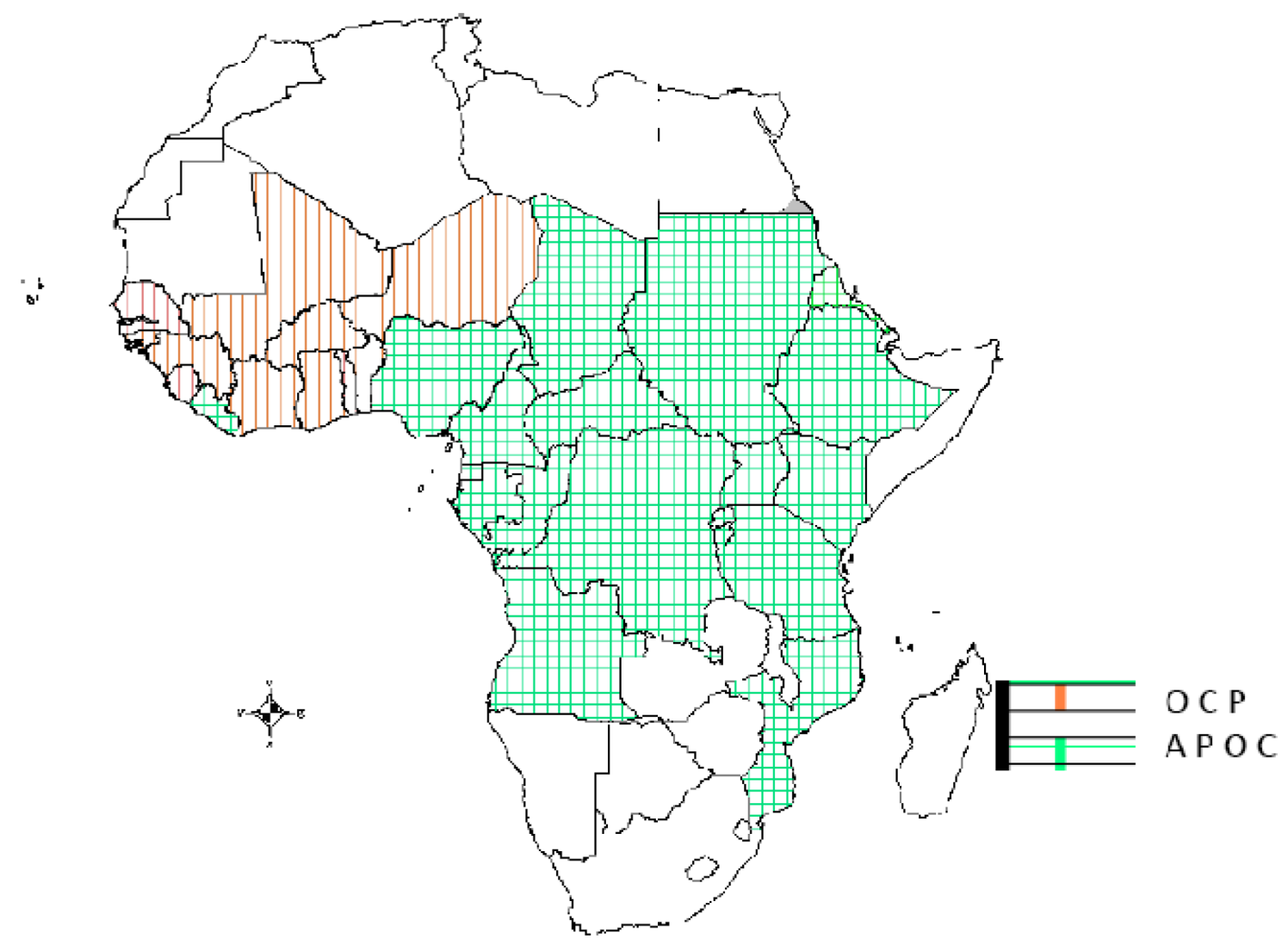

Figure I Map of Africa showing countries covered by the Onchocerciasis Control Project (OCP) and the African Onchocerciasis Control Programme (APOC). 


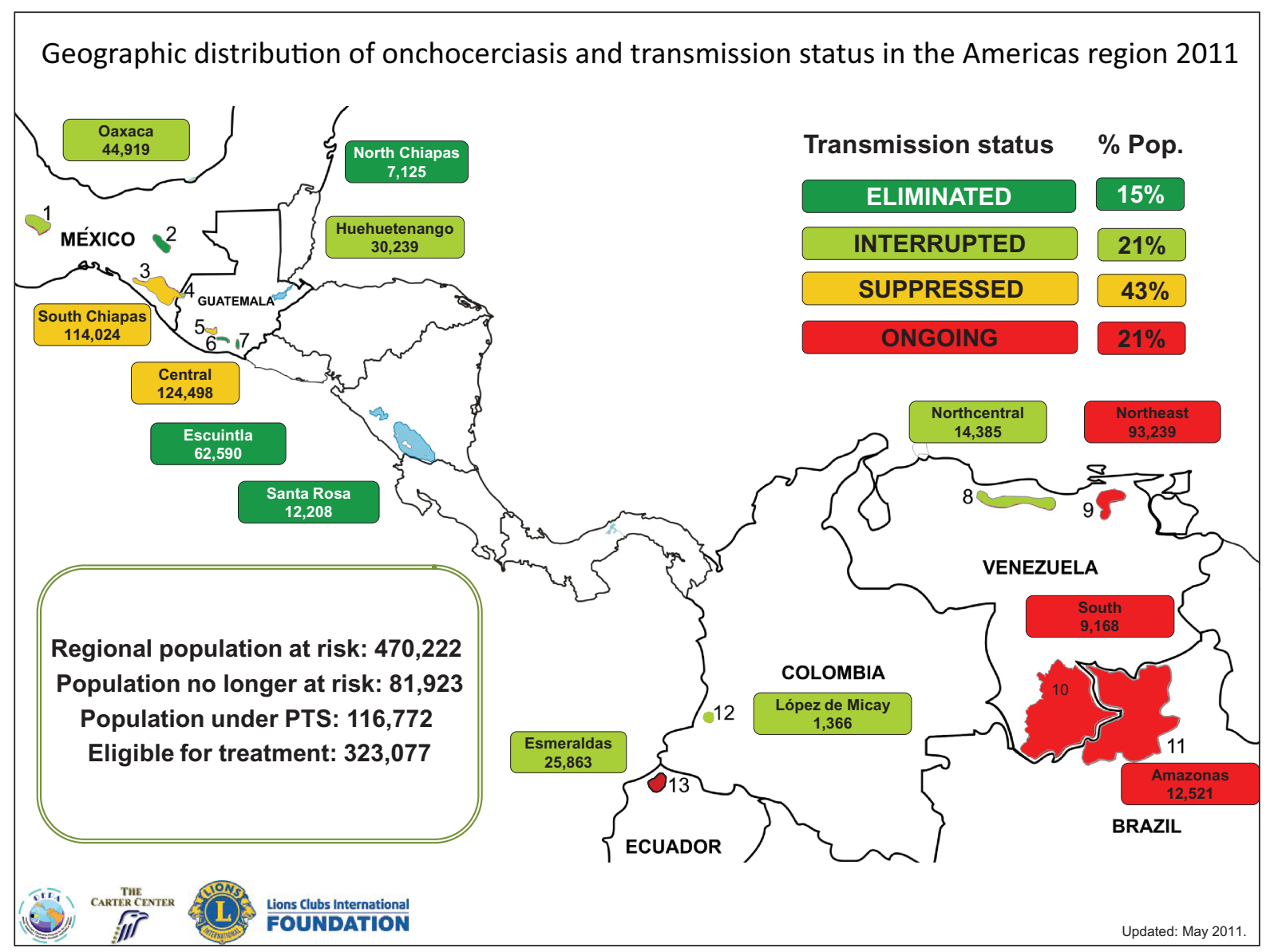

Figure 2 The Onchocerciasis Elimination Program for the Americas (OEPA).

Reproduced with permission from the Carter Foundation. Website http://www.cartercenter.org/resources/pdfs/health/river_blindness/OEPA-foci.pdf.

findings in onchocerciasis include corneal changes, such as sclerosing keratitis and snowflake opacities, torpid iritis characterized by typical pear-shaped deformity of the iris, secondary cataracts, choroidoretinopathy, and optic neuritis ${ }^{8}$ (see Figures 4 and 5).

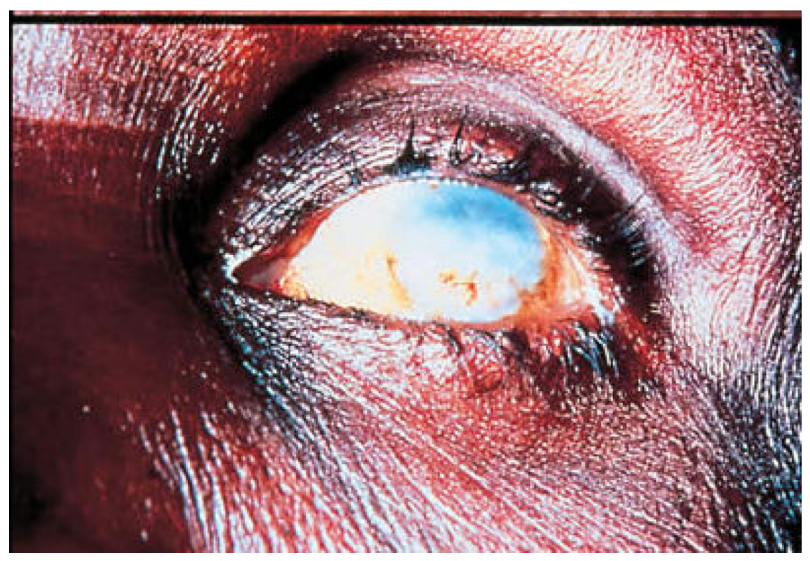

Figure 3 Sclerosing Keratitis in an African subject infested with Onchocerca volvulus.
The pathogenesis of optic neuritis is not well understood, but is believed to be associated with death of microfilaria and secondary inflammatory changes. ${ }^{9}$ The major causes of blindness in these patients are corneal blindness, particularly from sclerosing keratitis and chronic torpid iritis in the anterior segment, and chorioretinopathy and optic neuropathy in the posterior segment.

Most of the ocular changes have been attributed to direct infestation with microfilariae, particularly with the toxic reaction associated with their death. ${ }^{10}$ Massive and chronic death of the microfilariae is traditionally associated with sclerosing keratitis. However, evidence for this is not particularly strong. A paper examining the risk factors for sclerosing keratitis in the Kaduna State is underway. Death of individual microfilaria within the stroma of the cornea is associated with snowflake opacities. ${ }^{10}$ These lesions are potentially reversible with appropriate therapy. Some hold that sclerosing keratitis is also reversible but, in the author's experience, this is probably optimistic and debatable, and has yet to be clearly demonstrated. ${ }^{11}$ It is possible that, in addition 


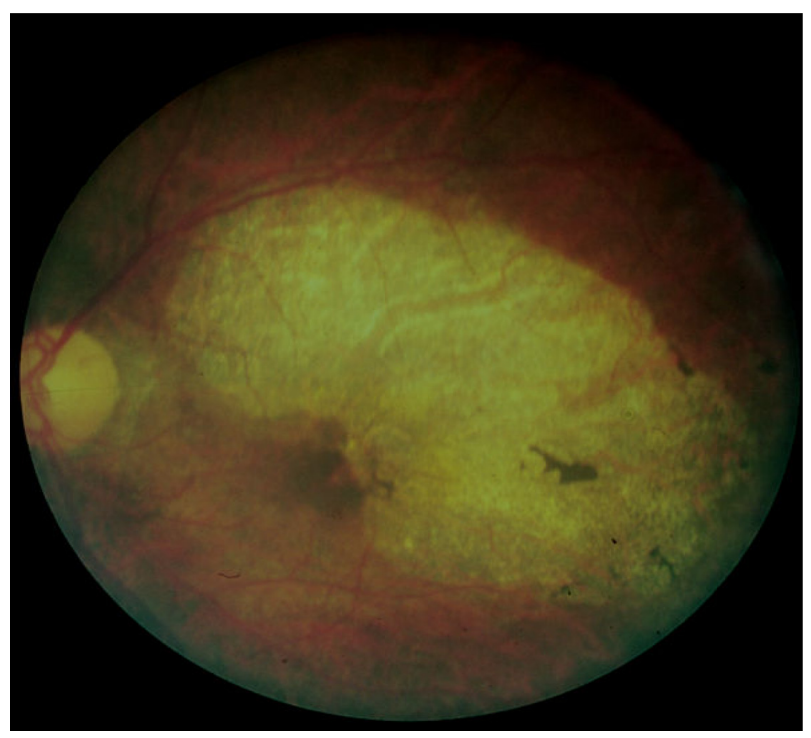

Figure 4 Typical optic atrophy and chorioretinal and pigment epithelial disease in a subject infested with Onchocerca volvulus.

to direct death of microfilariae, cross-reacting antigens may play a role in anterior segment lesions. ${ }^{12}$

The pathogenesis of retinochoroidal changes in onchocerciasis is still controversial. Researchers have investigated whether or not this is due to autoimmune reactions involving cross-reacting antigens between $O$. volvulus and interphotoreceptor retinoid-binding proteins and immune complex deposition pigment epitheliopathy, but there is no consensus on this. ${ }^{12-14}$

The management of ocular onchocerciasis needs to be evaluated from the level of vector management, management at the community level, and management at the individual level, including medical and surgical management.

\section{Vector control}

Various species of the anthropophilic blood-sucking blackfly are involved in the transmission of onchocerciasis.

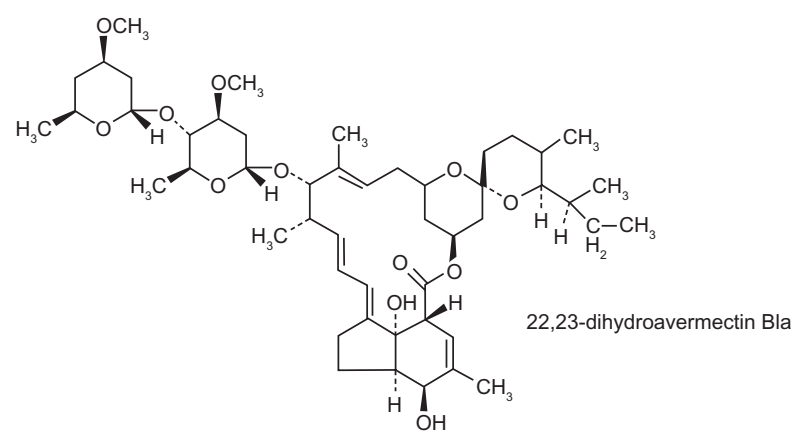

Figure $\mathbf{5}$ The chemical structure of ivermectin.
The most common vector in the West African subregion is Simulium damnosum. However, it must be noted that Simulidae are almost universally distributed, even in areas where onchocerciasis is not endemic, such as in Scotland. ${ }^{15}$ Measures have been taken to control the vectors, particularly in the OCP area of West Africa (1975-2002). ${ }^{16}$ This was a multilateral undertaking involving funding from the World Bank, the WHO as the executing agency, and the Food and Agricultural Organization and United Nations Development Agency taking the lead in development planning. The program aimed to eradicate onchocerciasis as a disease of public health concern in the eleven OCP countries west of Nigeria. The life cycle of $O$. volvulus can span 15 years until the natural death of the adult worm. Therefore, it is necessary to keep up vector control efforts for at least that period of time. The OCP, headquartered in Ouagadougou, interrupted transmission significantly in the target areas. ${ }^{17}$ For example, data from a Republic of Guinea focus suggest that before vector control measures, the mean annual transmission potential was about 120 microfilariae per person in 1975 . By 1985, following annual larviciding, this had dropped to less than five per person. Also, the number of infective flies and larvae per 1000 parous blackflies dropped from a peak of 80 and 50, respectively, to less than 10 . These levels are considered insufficient to sustain transmission. ${ }^{18}$ Larviciding was carried out using fixed wing planes and helicopters, as well as manual means where applicable. The initial chemical used was dichloro diphenyl trichloroethane (DDT). However, due to the environmental impact of this chemical, particularly its prolonged residence time, temephos $\left(\mathrm{Abate}^{\circledR}\right)$ which has a better environmental profile, was introduced and has been in use since. ${ }^{19}$

The OCP intervention has caused a significant reduction in the incidence of blindness from about $5 \%$ in the hyperendemic areas of the Upper Volta to $1 \%$ for all causes of blindness, while incident blindness from onchocerciasis appears to be no longer a problem. ${ }^{19}$ However, even in the treated areas of the OCP, there remain stubborn pockets of transmission requiring further vigilance. ${ }^{20}$

The OCP intervention is also deemed to be cost-effective. It is estimated that the total number of years of healthy life added by preventing blindness over the 20-year life of the project is $1,098,095 .{ }^{21}$ The overall cost of the project in 2011 would be about US\$100 million. Vector control is now applicable in selected areas, and is no longer regarded as the primary means of control of onchocerciasis. However, it should be noted that, in 1989, ivermectin distribution was also introduced into the OCP areas. $^{18}$ 


\section{Historical management of onchocerciasis}

The treatment of onchocerciasis before 1990 was with a combination of diethyl carbamazine (DEC, also known as banucide) for the microfilaria, and suramin for the adult worm. Suramin is a drug developed by Oskar Dressel and Richard Kothe of Bayer, Germany, in 1916, and is still sold by Bayer under the brand name Germanin ${ }^{\circledR}$. Suramin is a synthetic drug also used in the treatment of sleeping sickness (African trypanosomiasis) and more recently in the management of prostatic cancer. ${ }^{22}$ For onchocerciasis, suramin is administered by a single weekly intravenous injection for six weeks. The dose per injection is $1 \mathrm{~g}$. The most frequent adverse reactions are nausea and vomiting. About $90 \%$ of patients develop an urticarial rash (like a nettle or poison ivy-type rash) that disappears in a few days without needing to stop treatment. There is a greater than $50 \%$ chance of damage to the adrenal cortex, but only a small proportion of patients will require lifelong corticosteroid replacement. It is common for patients to get a tingling or crawling sensation of the skin with suramin. Suramin will cause clouding of the urine which is harmless, and patients should be warned of this to avoid alarm. Kidney damage and exfoliative dermatitis occur less commonly. ${ }^{23,24}$ Suramin is also associated with hepatic and bone marrow toxicity, the Stephens Johnson syndrome (often unreported in the author's experience), and death. ${ }^{25,26}$ Of note, some reports link suramin with the development of optic atrophy. ${ }^{27}$

On the other hand, DEC is associated with the Mazzotti reaction. First described in $1948,{ }^{28}$ the Mazzotti reaction is a symptom complex which can be life-threatening, and is characterized by fever, urticaria, swollen and tender lymph nodes, tachycardia, hypotension, arthralgias, edema, and abdominal pain that occurs within seven days of treatment for microfilariasis. The phenomenon is so common when DEC is used for the treatment of onchocerciasis that a specific skin patch test was developed to confirm the diagnosis. The drug patch is placed on the skin, and if the patient is infected with the microfilariae of $O$. volvulus, localized pruritus and urticaria are seen at the application site.

The prevailing hypothesis to explain the Mazzotti reaction is the abrupt release of parasite-specific antigens during cell death that induces a proinflammatory response associated with eosinophil migration to and degranulation in the skin. Death from the Mazzotti reaction has been described. ${ }^{29} \mathrm{DEC}$ is also anecdotally associated with optic atrophy, possibly resulting from a transient optic neuritis..$^{30,31}$ Using fluorescent angiographic techniques, this transient neuritis was demonstrated by
Bird et al to occur within 10-14 days of ingestion of DEC. ${ }^{32}$ These problems made DEC unsuitable for use as a mass chemotherapeutic agent in the management of onchocerciasis.

\section{Nodulectomy}

Mass nodulectomies were also carried out, particularly in the Americas. This made sense because the variant of onchocerciasis in the Americas was often associated with a preponderance of head nodules, which theoretically made it more likely that ocular lesions would eventuate. However, the effectiveness of this strategy in the long term is questionable, given that a study in Ecuador showed a reappearance of nodules in hyperendemic areas. ${ }^{33}$

\section{Chemotherapy after 1990}

The serendipitous discovery of the microfilaricidal potential of ivermectin radically changed the prospect of onchocerciasis control by chemotherapy. Ivermectin is isolated from the soil-dwelling mold, Streptomyces avermectilis. This mold was discovered by Omura et al near a golf course in Ito, Japan, and sent to the Merck, Sharp, and Dohme laboratories in $1974 .{ }^{34}$ In 1981 , ivermectin was marketed as a broad-spectrum anthelmintic drug in veterinary medicine, particularly for the dog heart worm Dirofilaria immitis. In 1982, researchers at Merck, Sharp, and Dohme reported activity against $O$. volvulus. ${ }^{35}$ The immediate concern then was whether or not ivermectin caused optic neuritis and secondary optic atrophy, as did DEC. A randomized controlled trial carried out in Kaduna State compared ivermectin with placebo and found that there was a significant difference in the incidence of optic neuritis between the two groups. ${ }^{36}$ The incidence ratio of optic neuritis (ivermectin vs placebo) was 0.90 (95\% confidence interval [CI]: 0.54-1.51) for subjects with microfilarial loads of $0-10$ microfilariae per $\mathrm{mg}$ of skin and 0.52 (CI: 0.29-0.93) for subjects with more than 10 microfilariae per mg of skin. This suggested that ivermectin reduced the incidence of optic neuritis in subjects with loads above 10 microfilariae per $\mathrm{mg}$, but had little effect in those with lower loads. The implication was that sustained annual delivery of ivermectin could prevent a substantial proportion of onchocercal blindness. ${ }^{33}$ In the same trial it was established that ivermectin did not precipitate optic neuritis within 2 weeks of ingestion, as did DEC. ${ }^{37}$ This trial and others $^{38,39}$ established the safety and efficacy of ivermectin.

\section{Pharmacology of ivermectin}

Ivermectin is a macrocyclic lactone and a synthetic derivative of a group of drugs called the avermectins (see Figure 5). ${ }^{40}$ 
Streptomyces avermitilis produces avermectin, a series of eight related pentacyclic lactones that contain a disaccharide of oleandrose, a methylated deoxysugar. ${ }^{41,42}$ Avermectin and the related compounds, milbemycin and nemadectin, are potent anthelmintic compounds, which are used commercially in animal health care and agriculture. The semisynthetic derivatives, avermectin C22, C23 dihydroavermectin B1, and ivermectin, are widely used for the treatment of diseases caused by nematodes and arthropods in the veterinary and agricultural fields, respectively. This class of drugs was introduced in the early 1980s and is active against a wide variety of helminths in several domestic animals, including cattle, sheep, horses, dogs, and pigs. ${ }^{43-45}$ In particular, it was noted to be active against the microfilariae of the dog heart worm, D. imitis, but not against the adult worm. ${ }^{46,47}$ These drugs act as agonists of gamma aminobutyric acid, which is the neurotransmitter utilized by most susceptible helminths. ${ }^{48-51}$ By keeping the postsynaptic chloride ion channels open when they should be closed, postsynaptic junctional transmissions are essentially blocked, leading to paralysis of the helminths. ${ }^{52}$ The host mammals, although utilizing gamma aminobutyric acid as a central nervous system transmitter, are usually not adversely affected because the large lactone molecule does not cross the blood-brain barrier with any facility. ${ }^{53}$

Therefore, the obvious limitation of ivermectin is that it is only active against the microfilariae, and at best, has limited effect against the adult worm, mainly in reducing the fertility of the adult female, particularly in high doses. ${ }^{54}$ For these reasons, the primary focus is now on the development of a safe and effective macrofilaricidal drug capable of being used in mass distribution programs.

\section{Community-directed treatment with ivermectin}

Because ocular damage is mostly attributable to the microfilaria, it became necessary to find chemotherapeutic agents active against both the microfilaria and the adult worm (either singly or in combination). This is because the adult worms, often found coiled up in subcutaneous nodules, sometimes adjacent to deep muscular tendons, have a life span of about 10-15 years and continue to produce microfilaria.

With confirmation of the efficacy and safety of ivermectin as a microfilaricide, it became possible to plan a control program with ivermectin, particularly in the non-OCP countries, which include Nigeria and the onchocerciasis-endemic countries in central and east Africa, as well as countries in Central and South America. APOC was set up in 1995 to eliminate onchocerciasis as a disease of public health importance in
Africa, while the equivalent program for the Americas was the Onchocerciasis Elimination Program for the Americas which commenced earlier in 1992. Merck has promised to make ivermectin available for distribution free of charge for as long as needed. ${ }^{55}$ The dosage schedule was simplified to utilize height rather than weight, because scales are not always available. ${ }^{56}$ In 1998, Merck expanded its commitment to include elimination of lymphatic filariasis in African countries and in Yemen where lymphatic filariasis coexists with river blindness. The program currently reaches more than 110 million people each year and continues to grow annually. Since inception of the program, Merck has donated two billion mectizan tablets at an estimated value of $\$ 3$ billion. Community-based treatment programs for river blindness exist in more than 117,000 communities in 27 countries in Africa, five countries in Latin America, and Yemen.

At the core of the APOC and Onchocerciasis Elimination Program for the Americas strategy to eliminate the disease is community-directed treatment with ivermectin, which relies on active community participation to distribute ivermectin to people who need it. This successful strategy is now being extended to include delivery of other health interventions, such as insecticide-treated nets for malaria. A project involving this method can include up to several hundred communities. These communities can span a state, province, or an entire country. Communities eligible for inclusion are identified using rapid epidemiological mapping of onchocerciasis, rapid epidemiological assessment, and geographical information systems techniques. ${ }^{57}$

The project approach allows for a phased introduction of community-directed treatment with ivermectin in a country. Each project is supported by the country's ministry of health and a nongovernmental development organization in most countries. As of the end of 2007, APOC was running 108 projects in 16 countries, and over 127,000 communities were involved. ${ }^{58}$ In Nigeria, for instance, there are projects in 27 states, including the Federal Capital Territory.

With the APOC mandate due to end in 2015, every effort is being made to ensure that the substantial investments and progress made towards eliminating onchocerciasis in Africa are sustained for the future. By 2015, APOC aims to transfer full responsibility for onchocerciasis control to local ministries of health, to encourage the ministries to provide ongoing financial support, and to encourage nongovernmental development organizations to continue to play their critical role. The goal is to establish country-led systems capable of eliminating onchocerciasis as a public health problem in all endemic countries in Africa. The Yaoundé Declaration was signed by 
African ministers of health in 2006. It expresses a renewed commitment to work towards the elimination of onchocerciasis in all countries where it is a public health problem.

There are challenges to the execution of the communitydirected treatment with ivermectin projects as stated by Amazigo et al. ${ }^{59}$ These include timely collection of drugs, integration of community-directed treatment with ivermectin into existing primary health care services, strengthening of local health infrastructure, achievement of optimal treatment coverage, establishment and upscaling of community self-monitoring, designing and implementing operations research locally, ensuring the adequacy of communitydirected distributors, and increasing the involvement of local nongovernmental organizations. Others are the achievement of financial sustainability and cost-recovery systems and effective advocacy. Coverage so far has varied from place to place. The following are not eligible to receive ivermectin: children five years or younger, pregnant women, and the obviously sick. ${ }^{60}$ However, coverage is deemed satisfactory where $\geq 90 \%$ of the annual treatment objective is met, as is the case of communities in Uganda, ${ }^{61}$ the annual treatment objective being the number of persons at risk of onchocerciasis in any given area of community-directed treatment with ivermectin who are estimated to require treatment.

Adverse drug events have been reported with ivermectin, but these have been generally mild, except in areas where there are overlaps with Loa loa.$^{62}$ In Sierra Leone, 28 (32\%) of 87 patients had adverse reactions that required treatment with acetylsalicylic acid and antihistamines, but none of the observed adverse reactions were considered life-threatening. The incidence of the Mazzotti reaction during treatment of onchocerciasis with ivermectin runs at approximately $10 \%$, which is much lower than that seen with DEC. Even so, nearly one-quarter of patients treated with ivermectin develop isolated fever or itching without the remaining constellation of symptoms. Because of its better safety profile, ivermectin has supplanted DEC as the drug of choice for the treatment of onchocerciasis. ${ }^{63}$ A significant relationship was found between the frequency and severity of side effects and the degree of parasite infestation. ${ }^{64}$ In Nigeria, a later study indicated that most of the reactions were experienced in the first round of dosing, and the frequency reduced with subsequent rounds. The most serious side effect noted in that study was joint pains. ${ }^{65}$

\section{Side effects with coexistent loiasis}

Several cases of encephalopathy have been reported after ivermectin in people heavily infected with the microfilariae of L. loa (loiasis) ${ }^{66}$ The relative risk of developing marked or serious reactions was significantly higher when the L. loa load exceeded 8000 microfilariae per $\mathrm{mL}$. The risk is very high (odds ratio $>1000$ ) for loads above 50,000 microfilariae per $\mathrm{mL}$. Epidemiological surveys aimed at assessing the intensity of infection with L. loa microfilariae, now called RAPLOA (Rapid Assessment Procedures for Loiasis) are now being carried out by WHO. Pictures of the L. loa worm are shown to communities for recognition. Those communities with a high rate of recognition are presumed to have high endemicity of Loiasis. This assessment should be carried out before ivermectin is distributed for onchocerciasis control in areas where loiasis is endemic. The most affected areas at the moment are the Cameroons and adjacent central African countries. In communities at risk, monitoring procedures should be established and adhered to during community-based ivermectin treatment, so that people developing serious reactions may receive appropriate treatment. ${ }^{66}$

\section{Interruption of transmission}

Ivermectin is mainly a microfilaricide but, in addition, has the effect of suppressing production of microfilaria by adult worms. However, this means that treatment would need to be continued for at least 15 years, which is the projected length of life of the adult. It has been debated as to whether or not this can lead to interruption of transmission. One study suggests that interruption is not likely to happen. ${ }^{67}$

In all of the river basins studied, treatment with ivermectin sharply reduced the prevalence and intensity of infection. However, significant transmission is still ongoing in some basins after 10-12 years of ivermectin treatment. In other basins, transmission may have been interrupted, but this needs to be confirmed by indepth evaluation. In one mesoendemic basin, where 20 rounds of 4-monthly treatment reduced prevalence of infection to levels as low as $2 \%-3 \%$, there was significant recrudescence of infection within a few years after interruption of treatment. ${ }^{68}$ These studies were carried out in West Africa. However, there are some important differences with the situation in the Americas where, by and large, the blackfly vector may not be as efficient, and population migrations are not a critical factor as they are in Africa. ${ }^{69}$ Using biannual ivermectin dosing with a high coverage, it has been possible to interrupt transmission in the Oaxaca focus in southern Mexico. ${ }^{70}$ In Latin America, transmission has been interrupted in half of the original endemic foci. In addition, Columbia appears to be the first country to report a nationwide elimination of onchocerciasis in the Americas. ${ }^{69,70}$ However, within Africa, there are isolated reports of elimination using ivermectin, particularly in Senegal and Mali. ${ }^{71}$ 


\section{Resistance to ivermectin}

Some disturbing instances of resistance have been reported in Africa. ${ }^{72}$ Workers in Ghana found that in some communities adult female worms were nonresponsive or resistant to the antireproductive effects of multiple treatments with ivermectin. These workers confirmed resistance by examining microfilaria repopulation levels one year after treatment, worm burdens per nodule, the age structure of adult female worms recovered from nodules, and the reproductive status of adult female worms 90 days after ivermectin treatment. They concluded that, in some communities, there was a loss of the effect of ivermectin on the suppression of parasite reproduction using the parameters stated.

There is also anecdotal evidence that some communitydirected treatment with ivermectin programs in the North Eastern area of Nigeria have already adopted higher frequency treatment due to poor response of skin lesions to annual therapy. Should resistance become more commonplace, the whole platform of the mass drug administration program might be in jeopardy, especially in Africa.

\section{Need for newer microfilaricides and macrofilaricides}

The challenges of maintaining distribution over a prolonged period, especially with the proposed cessation of funding of APOC and Onchocerciasis Elimination Program for the Americas programs in 2015, the recrudescence of transmission even in the face of continued community-directed treatment with ivermectin, and the possibility of development of resistance to ivermectin in the future (if not already) all make it urgent and necessary to develop newer drugs. Also, where there are overlapping foci involving lymphatic filariasis and $L$. loa, drug combinations may be necessary to prevent development of serious sequelae, such as encephalopathy. There are several candidate drugs on the horizon, some of which are undergoing trials.

\section{Moxidectin}

Moxidectin (milbemycin B) is a semisynthetic derivative of nemadectin which is produced by fermentation of Streptomyces cyanogriseus. This Streptomyces was discovered in a soil sample from Australia in the late 1980s collected by an agronomist working for American Cyanamid Company. Moxidectin treats and controls some of the most common internal and external parasites by selectively binding to the glutamate-gated chloride ion channels in parasites. These channels are vital to the function of invertebrate nerve and muscle cells; when moxidectin binds to the channels, it disrupts neurotransmission, resulting in paralysis and death of the parasite. Preclinical data in animal models suggest that moxidectin may either sterilize or kill the adult worm. ${ }^{73,74}$ Ivermectin does not do that, even though repeated treatments reduce the reproductive capacity of the adult worms. Drug trials in humans have therefore begun with moxidectin. A trial is being conducted by the Special Programme for Research and Training in Tropical Diseases, an independent global program sponsored by the United Nations Children's Fund, the World Bank, and the WHO. The trial will be enrolling 15,000 people for the study at four sites in Ghana, Liberia, and the Democratic Republic of Congo. The programme will involve African investigators and institutions. ${ }^{75}$ Subjects participating in the study will be randomly assigned (in a 2 to 1 ratio) to receive one orally administered dose of either moxidectin or ivermectin. The aim is to have a treatment that can be administered once or twice a year to interrupt transmission of the disease. At the moment, ivermectin needs to be given once or twice a year for 11-15 years to ensure control of the disease. Researchers believe moxidectin has the potential to control the disease after six cycles of treatment. ${ }^{76}$ Animal studies of moxidectin show that side effects vary by animal species, and may be affected by the product formulation, application method, and dosage.

\section{Flubendazole}

Flubendazole is another candidate macrofilaricide for onchocerciasis field programs. ${ }^{77}$ It is a very efficacious macrofilaricide in a variety of experimental animals, with perhaps its most dramatic and relevant action being an ability to eliminate adult $D$. immitis completely from dogs after a single injection. It was developed by Janssen in the mid 1970s and is currently licensed in Europe for use as an anthelmintic for humans with intestinal nematodes. In a number of experimental filarial rodent models, flubendazole had shown essentially $100 \%$ efficacy as a macrofilaricide at reasonable doses and schedules. A trial in human onchocerciasis was carried out in Mexico in the early $1980 \mathrm{~s}^{78}$ with promising results. Previous tests against Brugia Pahangi suggested that flubendazole may be active against adult tissue-dwelling filaria, but not against the microfilaria. ${ }^{79}$

However, wider testing in humans was restricted at that time by problems associated with the parenteral route of administration and the relatively unsophisticated carrier agent used, about 30 years ago. In addition, the introduction of ivermectin lessened the urgency to replace DEC for onchocerciasis control with a new macrofilaricide. In its currently available oral formulation, flubendazole is licensed in 
Europe for intestine-dwelling parasites. It is effective in this way because it does not need to be taken up into tissue. For it to be effective in an oral formulation against tissue-dwelling parasites such as $O$. volvulus, new excipients will need to be developed. One such promising excipient is hydroxypropyl$\beta$-cyclodextrin. ${ }^{80}$ Recent tests suggest that the cyclodextrin formulation could be effective against tissue-dwelling helminth parasites. ${ }^{81}$ The Bill and Melinda Gates Foundation is supporting a group of scientists enigmatically called "DOLF" (Death of Onchocerciasis and Lymphatic Filariasis) based at Washington University (St Louis, MO). Among other activities, this group is carrying out clinical trials on candidate macrofilaricides, including a new flubendazole formulation, against onchocerciasis. ${ }^{82}$

\section{Albendazole}

Albendazole is one of the benzimidazole group of drugs that is somewhat useful in onchocerciasis control. Albendazole was first discovered at the SmithKline Animal Health Laboratories in 1972. It is only available as an oral formulation. It is a broad-spectrum anthelmintic effective against roundworms, tapeworms, and flukes of domestic animals and humans. Albendazole (donated by GlaxoSmithKline) is being used to treat lymphatic filariasis as part of efforts to stop transmission of the disease in conjunction with ivermectin in subSaharan Africa, and with DEC elsewhere in the world. It is not strictly speaking a microfilaricidal for $O$. volvulus, but does have significant chemosterilant properties. It is believed to potentiate the effect of the microfilaricidal properties of ivemectin, and when used in this combination, can reduce microfilariasis by up to $99 \%$ for a year. ${ }^{83,84}$

The WHO Global Program to Eliminate Lymphatic Filariasis works actively to combat lymphatic filariasis by developing alternative and effective mass drug administration regimens and strategies. It aims to eliminate lymphatic filariasis globally by 2020. A higher dose of albendazole or biannual treatment is being trialed. As stated, where there are overlapping areas of onchocerciasis endemicity, albendazole is used alongside ivermectin, and the potentiating effect becomes an advantage. ${ }^{85}$

\section{Antibiotics targeting Wolbachia}

The symbiotic relationship between Wolbachia and the adult worms has been well elucidated. ${ }^{86}$ Most filarial species that infect people are symbiotic with Wolbachia bacteria, which are essential for the growth, development, and survival of their nematode hosts. This is probably because the worms are dependent on the bacteria for heme synthesis. ${ }^{87}$ These endosymbionts contribute to the pathogenesis of inflammatory disease and are a target for doxycycline therapy, which has macrofilaricidal activity, improves pathologic outcomes, and is as effective as monotherapy. ${ }^{88-90}$

Doxycycline $100 \mathrm{mg} /$ day orally for 6 weeks targets endosymbiotic bacteria and results in long-term sterility of the adult female worms and in a corresponding absence of microfilariae. In Ghana, when ivermectin was given after doxycycline, it resulted in a strong and sustained (18-month) reduction of microfilariae loads to levels of less than 0.3 microfilariae per mg. This may be a useful adjunctive therapy to ivermectin. ${ }^{89}$

\section{Coterminal foci of filarial infection}

As already noted, it is not uncommon in the African setting and elsewhere for more than one type of filarial disease to exist in the same focus. In such situations, mass chemotherapy needs to be planned taking this into consideration. There are nine known filarial nematodes which use humans as their definitive host. ${ }^{91-93}$ These are divided into three groups according to the niche within the body that they occupy, ie, serous cavity filariasis, lymphatic filariasis, and subcutaneous filariasis. Lymphatic filariasis is caused by the Brugia malayi, Wuchereria bancrofti, and Brugia timori worms. As the name suggests, these worms tend to occupy the lymphatic system, including the vessels and nodes, and are associated with development of elephantiasis. Lymphatic filariasis is transmitted by different types of mosquitoes, such as the Culex mosquito, Anopheles mainly in rural areas, and Aedes, mainly in endemic islands in the Pacific. Subcutaneous filariasis is caused by L. loa (the African eyeworm), Mansonella streptocerca, O. volvulus, and Dracunculus medinensis (the guinea worm). These worms occupy the subcutaneous layer of the skin, in the fat layer. Serous cavity filariasis is caused by the worms Mansonella perstans and Mansonella ozzardi, which occupy the serous cavity of the abdomen. In all cases, the transmitting vectors are either blood-sucking insects (flies or mosquitoes), or copepod crustaceans in the case of Dracunculus medinensis.

The recommended regimen for treatment through mass drug administration is a single dose of the two medicines given together, ie, albendazole $400 \mathrm{mg}$ plus either ivermectin $150-200 \mu \mathrm{g} / \mathrm{kg}$ in areas where onchocerciasis (river blindness) is also endemic or DEC $6 \mathrm{mg} / \mathrm{kg}$ in areas where onchocerciasis is not endemic. These medicines clear microfilariae from the bloodstream and kill most of the adult worms. However, at present, as noted earlier, ivermectin treatment is best avoided 
in areas where L. loa is also endemic to avoid the serious side effects.

\section{Impact of therapy on skin disease}

Workers suggest that the prevalence and severity of reactive skin lesions decrease with the use of ivermectin; ${ }^{94}$ in particular itching, which is one of the most disturbing aspects of morbidity, is reduced in frequency and intensity. The effect of long-term treatment on more chronic lesions was recently investigated by Ozoh et al. ${ }^{95}$ Significant reductions were found in all grades of skin disease after about five years of ivermectin therapy.

\section{Nuisance value of the blackfly}

It must be borne in mind that mass drug administration does not get rid of the blackfly. Outside the OCP in West Africa, vector control has been carried out only in selected areas. The nuisance value of the blackfly must not be underestimated. For instance, in the Democratic Republic of the Congo, the nuisance of $S$. damnosum represents a real problem on the Inga Dam and the two neighboring health zones (Inga and Seke-Banza), particularly during the high water period of the river between October and December. A total of 163,823 persons live in the area. Since cessation of vector control activities three decades ago, the daily biting rate has increased from 10 to 13,000 bites/person/day. ${ }^{96}$ This had serious negative health, social, and economic effects on workers and their families, in particular on school children, impairing their performance at school. APOC and WHO subsequently raised money for larviciding activity to control this menace. Other APOC zones have not been so lucky. There is still a need for larviciding where the biting rate is higher than an acceptable threshold. ${ }^{96}$

\section{Impact of longitudinal treatment with ivermectin}

This is summarized in Table 1. The impact of mass drug administration has been felt in the endemic areas and has, from estimates in 2006, prevented 500,000 cases of blindness, treated 20 million cases of severe itching, and averted 500,000 disability-adjusted life years per annum. ${ }^{97}$ The OCP, on the other hand, is estimated to have prevented 600,000 cases of blindness and allowed the reclamation of 25 million hectares of arable land. ${ }^{97}$ When specific ocular parameters are assessed, some workers suggest that there is a reversal of early ocular lesions, particularly snowflake opacities. ${ }^{98}$ The incidence of optic neuritis is also significantly reduced. ${ }^{37,99}$ However, there is controversy over the impact on visual
Table I Impact of longitudinal treatment with mectizan on certain parameters

\begin{tabular}{|c|c|}
\hline Source & Finding \\
\hline APOC 1996-200597 & 20 million cases of severe itching prevented \\
\hline APOC $1996-2005^{97}$ & 500,000 cases of blindness prevented \\
\hline APOC 1996-200597 & $\begin{array}{l}500,000 \text { DALYs per year averted at US\$7 } \\
\text { per DALY }\end{array}$ \\
\hline APOC 1996-200597 & Economic rate of return of $17 \%$ \\
\hline Abiose et $\mathrm{al}^{36}$ & $\begin{array}{l}\text { Prevention of new cases of optic neuritis } \\
\text { and prevention of optic nerve disease }\end{array}$ \\
\hline Abiose $^{98}$ & $\begin{array}{l}\text { Regression of early ocular lesions, including } \\
\text { sclerosing keratitis }\end{array}$ \\
\hline Cousens et $\mathrm{al}^{99}$ & A reduction in incidence of visual field loss \\
\hline $\begin{array}{l}\text { Ejere et al } \\
(\text { cochrane review })^{100}\end{array}$ & $\begin{array}{l}\text { No statistically significant difference was } \\
\text { observed in any trial (reporting visual acuity } \\
\text { outcome) between ivermectin and placebo } \\
\text { groups for visual acuity loss; questions about } \\
\text { the effectiveness of ivermectin in preventing } \\
\text { visual acuity loss have not been answered by } \\
\text { best available evidence }\end{array}$ \\
\hline Kennedy et $\mathrm{al}^{101}$ & $\begin{array}{l}\text { No change in the prevalence of ocular } \\
\text { signs such as punctate keratitis, sclerosing } \\
\text { keratitis, iridocyclitis, optic nerve atrophy, } \\
\text { cataract, glaucoma, chorioretinitis }\end{array}$ \\
\hline
\end{tabular}

Abbreviations: APOC, African Program for Onchocerciasis Control; DALY, disability-adjusted life years.

acuity, with a Cochrane review suggesting that there is no effect on this parameter. ${ }^{100}$ This seems consistent with the findings of Kennedy et al who followed mectizan users for five years and found no changes in the prevalence of main ocular lesions in this cohort. ${ }^{101}$ Interestingly, in the same paper, they reported a reduction in the prevalence of blindness, but it was not clear how this came about. There are perhaps obscure epidemiologic reasons for this. Patients with established ocular lesions may not benefit from treatment, and their visual acuity will likely remain unchanged. However, a reduced incidence of ocular involvement is expected, which over time will impact the overall prevalence of ocular lesions and indirectly impact the prevalence of blindness and visual impairment from onchocerciasis.

\section{Conclusion}

Ivermectin monotherapy through mass drug administration has had some positive impact on skin and ocular morbidity and incident blindness, but these gains may be fragile because of the possibility of drug resistance, and the need for sustained treatment for up to 15 years. The outcome of ongoing randomized controlled studies of candidate microfilaricides and safe macrofilaricides are eagerly awaited. In addition, areas where vector control can be of particular use need to be identified. Overall, the prospects of control are high, but 
comprehensive global eradication may not be achievable with current tools.

\section{Disclosure}

The author reports no conflict of interest in this work.

\section{References}

1. Duke BO. The Epidemiology of Eye Disease. 2nd ed. Johnson JJ, editor London, UK: Arnold Hodder Headline Group; 2003:288-305.

2. Ndyomugyenyi R. The burden of onchocerciasis in Uganda. Ann Trop Med Parasitol. 1998;92(1):133-137.

3. Raybould JN, White GB. The distribution, bionomics and control of onchocerciasis vectors (Diptera: Simuliidae) in Eastern Africa and the Yemen. Tropenmed Parasitol. 1979;30(4):505-547.

4. World Health Organisation. Onchocerciasis and its control. Technical report series No 852. Available from: http://www.who.int/bookorders/ anglais $/$ detart $1 . j s p ?$ sesslan $=1 \& \operatorname{codlan}=1 \& \operatorname{codcol}=10 \& \operatorname{cod} c \mathrm{ch}=852$ Accessed September 20, 2011.

5. Amazigo U, Noma M, Bump J, et al. Chapter 15. Onchocerciasis -Disease and Mortality in Sub-Saharan Africa. 2nd ed. Washington, DC: World Bank 2006-NCBI Bookshelf. Bookshelf ID: NBK2287 PMID 21290648. pp. 1-11.

6. World Health Organisation. Technical report series No 752. Geneva 1987.

7. Murdoch ME, Hay RJ, Mackenzie CD, et al. A clinical classification and grading system of cutaneous changes in onchocerciasis. Br J Dermatol. 1993;129(3):260-269.

8. Newland HS, White AT, Greene BM, Murphy RP, Taylor HR. Ocular manifestations of onchocerciasis in a rain forest area of west Africa. Br J Ophthalmol. 1991;75(3):163-169.

9. Thylefors B, Tonjum AM. Visual field defects in onchocerciasis. $\mathrm{Br} J$ Ophthalmol. 1978;62(7):462-467.

10. Eezzuduemhoi DR, Wilson D, Sheppard JD Jr, et al. Ophthalmologic Manifestations of Onchocerciasis. Available at: http://emedicine. medscape.com/article/1204593-overview. Accessed July 17, 2011.

11. Thylefors B. Ocular onchocerciasis. Bull World Health Organ. 1978;56(1):63-73.

12. McKechnie NM, Braun G, Kläger S, et al. Cross-reactive antigens in the pathogenesis of onchocerciasis. Ann Trop Med Parasitol. 1993;87(6):649-652.

13. Van der Lelij A, Doekes G, Hwan BS, et al. Humoral autoimmune response against $\mathrm{S}$-antigen and IRBP in ocular onchocerciasis. Invest Ophthalmol Vis Sci. 1990;31(7):1374-1380.

14. Paganelli R, Ngu JL, and Levinsky RJ. Circulating immune complexes in onchocerciasis. Clin Exp Immunol. 1980;39(3):570-575.

15. Zahar AR. The ecology and distribution of black flies (Simulidae) in south-east Scotland. J Anim Ecol. 1951;20(1):33-62.

16. Le Berre R, Walsh JF, Philippon B, et al. The WHO Onchocerciasis Control programme: retrospect and prospects. Philos Trans R Soc Lond B Biol Sci. 1990;328(1251):721-729.

17. Hougard JM, Yaméogo L, Sékétéli A, Boatin B and Dadzie KY. Twenty-two years of blackfly control in the onchocerciasis control programme in West Africa. Parasitol Today. 1997;13(11): 425-431.

18. Guillet P, Seketeli A, Alley ES, et al. Impact of combined largescale ivermectin distribution and vector control on transmission of Onchocerca volvulus in the Niger basin, Guinea. Bull World Health Organ. 1995;73(2):199-205.

19. Jamnback H. Recent developments in control of blackflies. Annu Rev Entomol. 1973;18:281-304.

20. Molyneux DH, Davies JB. Onchocerciasis control: Moving towards the millennium. Parasitol Today. 1997;13(11):418-425.

21. Prost A, Prescott N. Cost-effectiveness of blindness prevention by the Onchocerciasis Control Programme in Upper Volta. Bull World Health Organ. 1984;62(5):795-802.
22. La Rocca RV, Danesi R, Cooper MR, et al. Effect of suramin on human prostate cancer cells in vitro. J Urol. 1991;145(2):393-398.

23. Anderson J, Fuglsang H. Further studies on the treatment of ocular onchocerciasis with diethylcarbamazine and suramin. Br JOphthalmol. 1978;62(7):450-457.

24. Kaur M, Reed E, Sartor D, Dahut W, Fizz WD. Suramin development: What did we learn? Invest New Drugs. 2002;20(2):209-219.

25. Anderson J, Fuglsang H, de C Marshall TF. Effects of suramin on ocular onchocerciasis. Tropenmed Parasitol. 1976;27(3):279-296.

26. Arlt W, Reincke M, Siekmann L, Winkelmann W, Allolio B. Suramin in adrenocortical cancer: limited efficacy and serious toxicity. Clin Endocrinol (Oxf). 1994;41(3):299-307.

27. Thylefors B, Rolland A. The risk of optic atrophy following suramin treatment of ocular onchocerciasis. Bull World Health Organ. 1979;57(3):479-480.

28. Francis H, Awadzi K, Ottesen EA. The Mazzotti reaction following treatment of onchocerciasis with diethylcarbamazine: clinical severity as a function of infection intensity. Am J Trop Med Hyg. 1985; 34(3):529-536.

29. Stingl D, Sting1 M. Leprosy, onchocerciasis, diethylcarbamazine and the Mazzotti reaction. Lepr Rev. 1982;53(4):317-318.

30. Bryceson AD. What happens when microfilariae die? Trans $R$ Soc Trop Med Hyg. 1976;70(5-6):397-401.

31. Bryceson AD, Warrell DA, Pope HM. Dangerous reactions to treatment of onchocerciasis with diethylcarbamazine. $\mathrm{Br}$ Med $\mathrm{J}$. 1977;1(6063):742-744.

32. Bird AC, el-Sheikh H, Anderson J, Fuglsang H. Changes in visual function in the posterior segment of the eye during treatment of onchocerciasis with diethylcarbamazine citrate. $\mathrm{Br} J$ Ophthalmol. 1980;64(3):191-200.

33. Guderian RH. Effects of nodulectomy in onchocerciasis in Ecuador Trop Med Parasitol. 1988;39 Suppl 4:356-357.

34. The ivermectin story. Available at: http://www.satoshi-omura.info/ ivermectin/ivermectin.html. Accessed July 16, 2011.

35. Omura S, Crump A. The life and times of ivermectin - a success story. Nat Rev Microbiol. 2004;2(12):984-989.

36. Abiose A, Jones BR, Cousens SN, et al. Reduction in incidence of optic nerve disease with annual ivermectin to control onchocerciasis. Lancet. 1993;341(8838):130-134.

37. Murdoch I, Abiose A, Babalola O, et al. Ivermectin and onchocercal optic neuritis: short-term effects. Eye (Lond). 1994;8(Pt 4): 456-461.

38. Pacqué M, Muñoz B, Greene BM, Taylor HR. Community-based treatment of onchocerciasis with ivermectin: safety, efficacy, and acceptability of yearly treatment. $J$ Infect Dis. 1991;163(2):381-385.

39. Campbell WC, Benz GW. Ivermectin: a review of efficacy and safety. J Vet Pharmacol Ther. 1984;7(1):1-16.

40. Barragry RB. A review of the pharmacology and clinical uses of ivermectin. Can Vet J. 1987;28(8):512-517.

41. Burg RW, Miller BM, Baker EE, et al. Avermectins, new family of potent anthelmintic agents: producing organism and fermentation. Antimicrob Agents Chemother. 1979;15(3):361-367.

42. Campbell WC, Fisher MH, Stapley EO, Albers-Schonberg G, Jacob TA. Ivermectin: a potent new antiparasitic agent. Science. 1983;221(4613):823-828.

43. Nolan J, Schnitzerling HJ, Bird P. Evaluation of the potential of systemic slow release chemical treatments for control of the cattle tick Boophilus microplus using ivermectin. Aust Vet J. 1981;57(11): 493-497.

44. McPherson WB, Cairns GC, Scott PC. Productivity studies with ivermectin in sheep and cattle in New Zealand. In: Leaning WHD, editor. Recent Developments in the Control of Animal Parasites. Proceedings of the MSD AGVET Symposium on Recent Developments in the Control of Animals Parasites, XXII World Veterinary Congress, Perth, Australia. 1983:174-191.

45. Anderson RR. The use of ivermectin in the horse-research and clinical observations. Compend Contin Educ Pract Vet. 1984;6:S516-S520. 
46. Campbell WC, Blair LS. Efficacy of avermectin against Dirofilaria immitis in dogs. J Helminthol. 1978;52:308-310.

47. Blair LS, Campbell WC. Efficacy of ivermectin against Dirofilaria immitis larvae in dogs 31, 60 and 80 days after infection. Am J Vet Res. 1980;41(12):2108.

48. Fritz LC, Wang CC, Gorio A. Avermectin B1a irreversibly blocks post synaptic potentials at the lobster neuromuscular junction by reducing muscle membrane resistance. Proc Natl Acad Sci U S A. 1979;76(4):2062-2066.

49. Mellin T, Busch RC, Wang CC. Postsynaptic inhibition of invertebrate neuromuscular transmissions by avermectin B1a. Neuropharmacology. 1983;22(1):89-96.

50. Pony SS, Wang CC. Avermectin B1a increases the number of GABA receptors in thoroughly washed rat brain membranes. Abstract presented at the 10th Annual Meeting of the Society for Neuroscience, Cincinnati, OH, June14-17, 1980.

51. Pony SS, Wang CC, Fritz LC. Studies on the mechanisms of action of avermectin B1a: stimulation of release of gamma amino butyric acid from brain synaptosomes. $J$ Neurochem. 1980;34(2):351-358.

52. McCavera S, Rogers AT, Yates DM, Woods DJ, Wolstenholme AJ. An ivermectin-sensitive glutamate-gated chloride channel from the parasitic nematode Haemonchus contortus. Mol Pharmacol. 2009;75(6): $1347-1355$

53. Doss R. Ivermectin Toxicity Signs. 2009. Available at: http://www. livestrong.com/article/32684-ivermectin-toxicity-signs/. Accessed on October 13, 2011.

54. Gardon J, Boussinesq M, Kamgno J, Gardon-Wendel N, DemangaNgangue, Duke BO. Effects of standard and high doses of ivermectin on adult worms of Onchocerca volvulus: a randomised controlled trial. Lancet. 2002;360(9328):203-210.

55. The Merck MECTIZAN ${ }^{\circledR}$ Donation Program: 20 Years of Progress. Available at: http://www.merck.com/corporate-responsibility/docs/ MDP-Backgrounder-2008.pdf. Accessed July 11, 2011.

56. Alexander ND, Cousens SN, Yahaya H, Abiose A, Jones BR. Ivermectin dose assessment without weighing scales. Bull World Health Organ. 1993;71(3-4):361-366.

57. Noma M, Nwoke BE, Nutall I, et al. Rapid epidemiological mapping of onchocerciasis (REMO): its application by the African Programme for Onchocerciasis Control (APOC). Ann Trop Med Parasitol. 2002;96 Suppl 1:S29-S39.

58. APOC WEBSITE. Available at: http://www.who.int/apoc/cdti/projects/ en/index.html. Accessed June 19, 2011.

59. Amazigo UV, Brieger WR, Katabarwa M, et al. The challenges of community-directed treatment with ivermectin (CDTI) within the African Programme for Onchocerciasis Control (APOC). Ann Trop Med Parasitol. 2002;96 Suppl 1:S41-S58.

60. Brieger WR, Otusanya SA, Oke GA, Oshiname FO, Adeniyi JD. Factors associated with coverage in community-directed treatment with ivermectin for onchocerciasis control in Oyo State, Nigeria. Trop Med Int Health. 2002;7(1):11-18.

61. Katabarwa MN, Habomugisha P, Richards FO Jr. Implementing community-directed treatment with ivermectin for the control of onchocerciasis in Uganda (1997-2000): an evaluation. Ann Trop Med Parasitol. 2002;96(1):61-73.

62. Rothova A, Stilma JS, Van Der LelijA, Wilson WR, Barbe RF. Side-effects of ivermectin in treatment of onchocerciasis. Lancet. 1989;1(8652):1439-1441.

63. Ottesen EA. Ivermectin in human Medicine. J Antimicrob Chemother. 1994;34(2):195-203.

64. Ivermectin Side Effects. Available at: http://www.drugs.com/sfx/ ivermectin-side-effects.html. Accessed on October 10, 2011.

65. Oyibo WA, Fagbenro-Beyioku AF. Adverse reactions following annual ivermectin treatment of onchocerciasis in Nigeria. Int $J$ Infect Dis. 2003;7(2):156-159.

66. Gardon J, Gardon-Wendel N, Demanga-Ngangue, Kamgno J, JeanChippaux JP, Boussinesq M. Serious reactions after mass treatment of onchocerciasis with ivermectin in an area endemic for Loa loa infection. Lancet. 1997;350(9070):18-22.
67. Borsboom G, Boatin B, Nagelkerke N, et al. Impact of ivermectin on onchocerciasis transmission: assessing the empirical evidence that repeated ivermectin mass treatments may lead to elimination/eradication in West-Africa. Filaria J. 2003;2(1):8. Available at: http://www. filariajournal.com/content/2/1/8. Accessed July 7, 2011.

68. Dadzie Y, Neira M, Hopkins D. Final report of the Conference on the eradicability of Onchocerciasis. Filaria J. 2003;2(1):2.

69. Rodríguez-Pérez MA, Unnasch TR, Domínguez-Vázquez A, et al. Interruption of transmission of Onchocerca volvulus in the Oaxaca focus, Mexico. Am J Trop Med Hyg. 2010;83(1):21-27.

70. Murdoch ME. Onchodermatitis. Curr Opin Infect Dis. 2010;23(2): 124-131.

71. Diawara L, Traoré MO, Badji A, et al. Feasibility of onchocerciasis elimination with ivermectin treatment in endemic foci in Africa: first evidence from studies in Mali and Senegal. PLoS Negl Trop Dis. 2009;3(7):e497.

72. Osei-Atweneboana MY, Awadzi K, Attah SK, Boakye DA, Gyapong JO, Prichard RK. Phenotypic evidence of emerging ivermectin resistance in Onchocerca volvulus. PLoS Negl Trop Dis. 2011;5(3):e998.

73. Cotreau MM, Warren S, Ryan JL, et al. The antiparasitic moxidectin: safety, tolerability and pharmacokinetics in humans. J Clin Pharmacol. 2003;43(10):1108-1115.

74. A suitable macrofilaricide? Available at: https://apps.who.int/tdr/ publications/tdrnews/news62/moxidectin.htm. Accessed July 12, 2011.

75. A single-dose, ivermectin-controlled, double-blind, efficacy, safety and tolerability study of orally administered moxidectin in subjects infected with onchocerca volvulus. Available at: http://clinicaltrials. gov/ct2/show/NCT00790998. Accessed July 10, 2011.

76. N Siva. WHO researchers start trial on a new drug for river blindness BMJ. BMJ. 2009;339:b2755. Available at: www.bmj.com/content/339/ bmj.b2755.extract. Accessed on October 10, 2011.

77. Mackenzie CD, Geary TG. Flubendazole: a candidate macrofilaricide for lymphatic filariasis and onchocerciasis field programs. Expert Rev Anti Infect Ther. 2011;9(5):497-501.

78. Dominguez-Vasquez A, Taylor HR, Greene BM, et al. Comparison of flubendazole and diethylcarbamazine in treatment of onchocerciasis. Lancet. 1983;1(8317):139-143.

79. Denham DA, Samad R, Cho SY, Suswillo RR, Skippins SC. The anthelmintic effects of flubendazole on Brugia pahangi. Trans $R$ Soc Trop Med Hyg. 1979;73(6):673-676.

80. Challa R, Ahuja A, Ali J, Khar RK. Cyclodextrin in drug delivery: an updated review. AAPS PharmSciTech. 2005;6(2):E329-E357.

81. Ceballos L, Elissondo M, Bruni SS, Denegri G, Alvarez L, Lanusse C. Flubendazole in cystic echinococcosis therapy: pharmaco-parasitological evaluation in mice. Parasitol Int. 2009;58(4):354-358.

82. Death to Onchocerciasis and Lymphatic Filariasis [hompage]. Available at: http://www.dolf.wustl.edu/. Accessed June 24, 2011.

83. Mectizan and Albendazole for LF. Available at: http://www.mectizan. org/mectizan-albendazole-lf. Accessed July 12, 2011.

84. Awadzi K, Hero M, Opoku NO, et al. The chemotherapy of onchocerciasis XVII. A clinical evaluation of albendazole in patients with onchocerciasis; effects of food and pre-treatment with ivermectin on drug response and pharmacokinetics. Trop Med Parasitol. 1994;45(3):203-208.

85. Bockarie MJ, Deb RM. Elimination of lymphatic filariasis: do we have the drugs to complete the job? Curr Opin Infect Dis. 2010;23(6): 617-620.

86. Hoerauf A, Volkmann L, Hamelmann C, et al. Endosymbiotic bacteria in worms as targets for a novel chemotherapy in filariasis. Lancet. 2000;355(9211):1242-1243.

87. Wu B, Novelli J, Foster J, et al. The heme biosynthetic pathway of the obligate wolbachia endosymbiont of brugia malayi as a potential anti-filarial drug target. PLos open access library. Available at: http:// www.plosntds.org/article/info\%3Adoi\%2F10.1371\%2Fjournal. pntd.0000475. Accessed July 20, 2011.

88. Taylor MJ, Hoerauf A, Bockarie M. Lymphatic filariasis and onchocerciasis. Lancet. 2010;376(9747):1175-1185. 
89. Slatko BE, Taylor MJ, Foster JM. The Wolbachia endosymbiont as an anti-filarial nematode target. Symbiosis. 2010;51(1):55-65.

90. Hoerauf A, Mand S, Volkmann L, et al. Doxycycline in the treatment of human onchocerciasis: kinetics of Wolbachia endobacteria reduction and of inhibition of embryogenesis in female Onchocerca worms. Microbes Infect. 2003;5(4)261-273.

91. Filarial worms Database. Available at: http://www.broadinstitute.org/ annotation/genome/filarial_worms/MultiHome.html. Accessed July 17, 2011.

92. Darben P. The filarial nematodes. 1997 [updated January 31, 2002]. Available at: http://home.austarnet.com.au/ wormman/WLNEMA1. HTM. Accessed on October 8, 2011.

93. Filariasis. Available at: http://www.who.int/topics/filariasis/en/Accessed July 17, 2011.

94. Brieger WR, Awedoba AK, Eneanya CI, et al. The effects of ivermectin on onchocercal skin disease and severe itching: results of a multicentre trial. Trop Med Int Health. 1998;3(12):951-961.

95. Ozoh GA, Murdoch ME, Bissek AC, et al. The African Programme for Onchocerciasis Control: impact on onchocercal skin disease. Trop Med Int Health. 2011;16(7):875-883.
96. African Programme for Onchocerciasis Control. Available at: http:// www.who.int/apoc/publications/progress_report_jaf13_2007_en.pdf. Accessed July 7, 2011

97. Onchocerciasis control partners' meeting. A strategic overview of the future of onchocerciasis control in Africa. WHO/APOC; 2006. Available at: http://www.who.int/apoc/publications/final_report_of the_working_group_en.pdf. Accessed July 7, 2011.

98. Abiose A. Onchocercal eye disease and the impact of Mectizan treatment. Ann Trop Med Parasitol. 1998;92 Suppl 1:S11-S22.

99. Cousens SN, Cassels-Brown A, Murdoch I, et al. Impact of annual dosing with ivermectin on progression of onchocercal visual field loss. Bull World Health Organ. 1997;75(3):229-236.

100. Ejere H, Schwartz E, Wormald R. Ivermectin for onchocercal eye disease (river blindness). Cochrane Database Syst Rev. 2001;(1):CD002219.

101. Kennedy MH, Bertocchi AD, Hopkins AD, Meredith SE. The effect of 5 years of annual treatment with ivermectin $\left(\right.$ Mectizan $\left.^{\circledR}\right)$ on the prevalence and morbidity of onchocerciasis in the village of Gami in the Central African Republic. Ann Trop Med Parasitol. 2002;96(3):297-307.
Clinical Ophthalmology

\section{Publish your work in this journal}

Clinical Ophthalmology is an international, peer-reviewed journal covering all subspecialties within ophthalmology. Key topics include: Optometry; Visual science; Pharmacology and drug therapy in eye diseases; Basic Sciences; Primary and Secondary eye care; Patien Safety and Quality of Care Improvements. This journal is indexed on

Submit your manuscript here: http://www.dovepress.com/clinical-ophthalmology-journal

\section{Dovepress}

PubMed Central and CAS, and is the official journal of The Society of Clinical Ophthalmology (SCO). The manuscript management system is completely online and includes a very quick and fair peer-review system, which is all easy to use. Visit http://www.dovepress.com/ testimonials.php to read real quotes from published authors. 